$$
\cos t-7207 / 7--2
$$

\title{
COMPUTER CONTROL SYSTEM FOR ORIC*
}

C. A. Ludemann, J. M. Domaschko, ${ }^{+}$and S. W. Mosko

Oak Ridge National Laboratory, Oak Ridge, Tennessee 37830

\section{ABSTRACT}

A computer control system is being Implemented for the Oak RIdge Isochronous Cyclotron (ORIC). At the start of a run, operating parameters are set automatically using data stored in a disc 11brary. Adjustments of these parameters are made through an operator-computer interface consisting of a CRT unit with a keyboard. An array of assignable push-button switches is available for fine tuning various machine parameters. ORIC has 43 ungrounded power supplies requiring adjustment resolution to within 1 part in $10^{3}$ to 1 part in $10^{3}$ depending upon their function. These supplies are controlled by reference voltages derived from 12to 16-bit digital-to-analog converters (DAC's). A programmable comparator associated with each power sipply relays information on power supply performance to the computer. The power supplies, DAC' 8 , and comparators are isolated from the conputer by optical couplings and blocking capacitors. The control system sets and fine tunes the rf system, monitors and controls binary functions, swltches power supplies to required circuits, alerta the operator if any cyclotron parameter exceeds the opecified 1imits, and processes and stores new run parameters.

\section{INTRODUCTION}

Computerized control systems are considered as standard equipment for many new accelerators. For existing facilities, the expense of converting to computerized controls is justified only if it will result in improved machine utilization and performance. ORIC has always been a rather complex facility and it is becoming more complex with the addition of new external and internal ion sources, 1,2 new experimental areas, and an isotope separator (UNISOR). Also in the National Heavy-Ior. Laboratory (NHL) ${ }^{3}$ Proposal ORIC is included as

\footnotetext{
* Research sponsored by the United States Atomic Energy Comiseion under contract with Union Carbide Corporation.
}

tyationel science Poundation Presidential Intern. 
an infictor. Computer control wlll be essential for such an operation. Converting ORIC to computerlzed controls willi save hundreds of liours in set-up and malntenance time. This time will be used in productive research activity. In addition, experiments requiring frequent energy changes will become practical, and the operating stafl will be able to provide more assistance to users.

The set-up routine at ORIC typically involves setting a large number of parameters at values logged on previous runs. As a run gets underway, an assortment of component malfunctions are of ten discovered through their preventing normal beam extraction and transmission. Data interpretation errors often result in prolonged set-up time.

The computerized control system planned for ORIC automatically selects power supply polarities and output settings. It pretunes the rf system and performs a multitude of "switching on" operations. Parameters for a given Ion beam are read from the computer's disc library and the computer interpolates between sets of data where necessary. Automatic diagnostic routines locate and evaluate faulty components. Convenient, rellable bookkeeping is provided for recording useful operating parameter data and upgrading the disc 11brary as requi red.

An instant changeover to computerized controls would be convenlent, not withstanding operator consternation; however, computerization will be a gradual process. Interfacing the computer to the cyclotron 18 fairly simple for most analog instrumentation and controls, but implementing contact closure functions will require rewiring portions of the present control system. Long range plans include automatic compensating adjustments on several parameters as one or more other parameters are being optimized. Automatic beam optimization will also be considered.

\section{AN INVENTORY OF ORIC CONTROLS}

An approximate inventory of existing instrumentation and controls is given in Table $\mathrm{I}$. High resolution implies nominally $1 \mathrm{pp} 10^{3}$, medium resolution -1 pp $10^{4}$, and low resolution $-1 \mathrm{pp} 10^{3}$ or less. Analog references are required for setting power supply output levels and pre-setting tuners in the rf system. Many of the devices considered here are already "remotely programmed" via potentiometer controlled reference voltages. "Contact closures" include many functions from push button and toggle switches on the control console to trim coll polarity reversing switches and quadrupole selection switches.

High resolution analog data are digital voltmeter readings representing currents in each of the magnet windings. The main fleld, trim colls, valley colls, harmonic colls, and beam optics are Included. Low resolution analog data 18 presently acquired from a multitude of ponel wetero in assorted alzes, shapes, and 
callbrations. Contact sensing is presently accomplished through approprlate sensing switches with readout via pllot lights, flaghing lights, bells, and buzzers in the control room.

Table I ORIC Instrumentation and Controls Inventory

\begin{tabular}{lr}
\hline High resolution analog reference & 3 \\
Medium resolution analog references & 80 \\
Contact closures & 240 \\
High resolution analog data & 46 \\
Low resolution analog data & 110 \\
Contact sensors & 425 \\
\hline
\end{tabular}

THE CONTROL COMPUTER AND PERIPHERALS

Table II 1 ists the components of the computer system which have been ordered for ORIC. Delivery of the equipment 18 expected during the fall of 1972 .

\section{Table II Control Computer and Peripherals}

A general purpose computer, 16 blts/word, $24 \mathrm{~K}$ wemory. An operator keyboard/printer.

A high speed paper tape reader.

A high speed paper tape punch.

A magnetic tape drive.

A disc storage device.

A card reader.

A high speed serial printer.

A CRT graphic and alphanumeric dieplay with semiconductor memory and keyboard.

A digital input/output subsystem.

An 80-channel, multiplexed input, analog-to-digital converter/sys tem.

A library of operating parameters for vartous lons and energies will be kept on the storage diac. The main operator-computer interface will be through the CRT graphic terminal and keyboard. Future plans include a touch actuated syste for the CRT display, but for the present time, direct interaction with the display will be via a cursor and the keyboard. A set of asaignable push-button controls will pernit oinulteneou fine tuning of everal selected paranetera. 
Before start-up, the operator will call for a index of run parameters in the library, and select an approprlate reference run. When commanded, the computer will begin 1 ts set-up program. The computer will continually monitor the many survel1lance channels and alert the operator if any component requires special attention.

Interfacing between the computer and the cyclotron wi11 be through 23 digital input-output channels (16 bits each) in the I/O subsystem. The 80 channel ADC unit will scan and read all parameters which are presently on panel meters. An existing 6 digit DVM and crossbar scanner which scans all power supplies and several position Indicator devices will be interfaced to the computer to provide high resolution input data. Interfacing for the analog output channels and binary I/0 channels will be via dacaways as in the CAMAC system.

\section{POHER SUPPLY CONTROL AND SURVEILLANCE}

The first stage in implementing the computerized control system is automatic set-up and survelilance of the 43 magnet power supplies. The central processing unit, CPU, transmits digital data to a digital-to-analog converter, DAC, assoniated with each power supply. The DAC generates an analog reference signel suitable for controlling its respective power supply. A survel.llance device informs the computer whether or not the power supply is responding correctly to its instructions.

Numerous DAC schemes are avallable including stepping motor actuated potentiometers and relay switched ladder networks. At ORIC we are using nicrocircuit DAC's which offer excellent performance and are available at very low cost. Twelve bit DAC's are used for most power supplies and 16-bit DAC's for those requiring espectally high resolution as on the maln fleld and the $153^{\circ}$ beam analyzing magnet.

The use of survelllance devices and microcircuit DAC's is complicated by the electrical isolation requirements for each power supply. At ORIC, all magnet windings and power supplies are insulated from ground and from each other. Consequently, all associated circuitry requires common mode insulation ratings up to $400 \mathrm{~V}$. Optical coupling is suitable for transmitting logic levels, and capacitor or trans former coupling may be used for transmitting pulses between the CPU and the power supplies. Analog input information is best handled by analog-to-digital conversion prior to transmission.

A computerized control system is especially useful if it provides continuous survelilance of all power supplies. It should be able to resolve errors in poser supply regulation of a few tenths of one percent and to detect excessive ripple at frequencies up to $360 \mathrm{~Hz}$ or oscillation at frequencies up to a few $\mathrm{kHz}$. A digltal voltweter with multiplexer cen provide oufficient information if it has a copling rate of at least 10,000 eamples/sec and at least 
12 bit resolution. Such devices are available; however, they are typically single -ended or otherwise unable to withstand common mode potential greater than a few volts.

The possibility of providing a microcircuit analog-digttalconverter, ADC, for each power supply was considered, but an examination of how an ADC works led to a less complicated and more economical device. Since an ADC typically consists of a DAC and a voltage comparator and a DAC 18 already belng provided for each power supply, the addition of a comparator results in a gystem which generates all the required performance information.

A power supply control loop Incorporating DAC's and comparators is shown in $\mathrm{FIg} .1$. When a shange in magnet current is required, the CPU generates a "data word" representing the magnitude of the change and an "address word" representing which power supply is to be changed and related instructions. The data vord is converted to a series of pulses and routed to the power supply controller by a decoder which is controlled by the address word register. Conversion is accomplished by loadin $n_{i}$ the data word into an intermediate UP/DOWN counter. A NOR gate, "clear indicator," senses the non-zero s tate of the counter, Informs the CPU that the loop 18 "busy," and opens the "pulse gate." As pulscs are transmitted to the decoder, the intermediate counter counts down. When the counter reaches zero, the clear indicator closes the pulse gate and informs the CPU that the loop ts ready for more data.

There are three DAC's associated with each power supply. Each DAC is controlled by an UP/LOWN counter. Each UP/DOWN counter has inputs for count up, count down, and reset. The power supply DAC provides a reference signal for the power supply regulator. An analog signal fron the power supply shunt is inverted and compared to the DAC voltage at the suming point on the input of the two differential comparators. For normal conditions both comparators, monitored by the CPU, have low logic levels. If the power supply current is too high, the upper limit comparator switches to a high logic level where it remains as long as this condition persists. Likewise, the lower limit comparator switches to a high logic level if the power supply current is too low. The trigger points for the comparators are programable through of fset signals derived from the upper limt and lower limit DAC's, respectively.

Normally, the comparators are set for some predetermined tolerance. If the tolerance is exceeded, the $\mathrm{CPU}$, alerted through an interrupt, locates the faulty unit by scanning 1 ts input channels, and transmits a warning to the operator. If desired, the operator initiates a diagnostic routine where by the CPU 8 cans the comparators as a function of tine to evaluate ripple and noise structure if any. Then the CPU raises the comparator thresholds oufficiently to keep then at 10 i logic levels so that the limit DAC's settings represent either peak to peak ripple and noise level or a power supply tracking error. An actual display of data taken for a power oupply with 
excessive ripple is shown in Fig. 2. The display is composed of three sets of comparator scans with the comparators checked at 1 millisecond intervals. The D $\Lambda C$ settings are shown at the beginning of each set. The symbol "I" Indicates power supply within tolerance. The symbol "*" Indicates power supply high or low depending upon whether it is left or right of center. The time scale for each set of scans is printed for scans which indicate a change in logic levels since the preceeding scan. The scan rate 18 varlable as needed for resolving noise structure. Frequencles of several $\mathrm{kHz}$ can be resolved.

One data-to-pulse conversion unit may be used for controlling several power supplies through approprlate decoder addressing. The repetition rate of the pulse generator 18 fired at some value compatable with the slewing rate of the power supplies. While the conversion unt is busy, the CPU is avaliable for other tasks.

The CPU will periodically check the existing DVM and crossbar ocanner for calibration purposes.

\section{STATUS OF THE CONTROL SYSTEM}

The computer and its peripherals have been ordered. Delivery is expected during the fall of 1972 . A single power supply control channel has been assembled and tested using the on-line data processing system, an SEL 840A, as a CPU. One of the trim colls was operated for two days using computer control.

Low resolution analog signals which are presently displayed by panel meters are imuediately available for the computer's ADC systen. Control unt ts w111 be fabricated for 43 magnet power supplies and installed early in 1973. The next stage of the program includes interfacing the computer with the power supply polarity and quadrupole selection switch gear. Later stages will include hardware for pretuning the rf system and operating the ion sources.

Initial control software will be developed with sufficient flexability for incorporating software additions and changes required with later stages of hardware development. Adepting computer control to more difficult problens ouch as beam optindzation and diagnostic routines will offer a continuing software challenge.

\section{RE FERENCES}

1. ORNL Electronuclear Div. Annu. Progr. Rept., 12/31/68, ORNL 4404, pp. 96-101.

2. E. D. Hudson, M. L. Mallory, and S. W. Mosko, High Performance Heavy-Ion Sources for Cyclotron, IEEE Nuc. Sci. Trans. NS-18, No. 3. (1971) p. 113.

3. J. A. Martin, E. D. Hudson, R. S. Lord, M. L. Mallory, W. T. Milner, S. W.Kosko, and P. H. Stelson, A Proposed National HeavyIon Accelerator at Oak Ridge, Proceedinge of this Conference. 


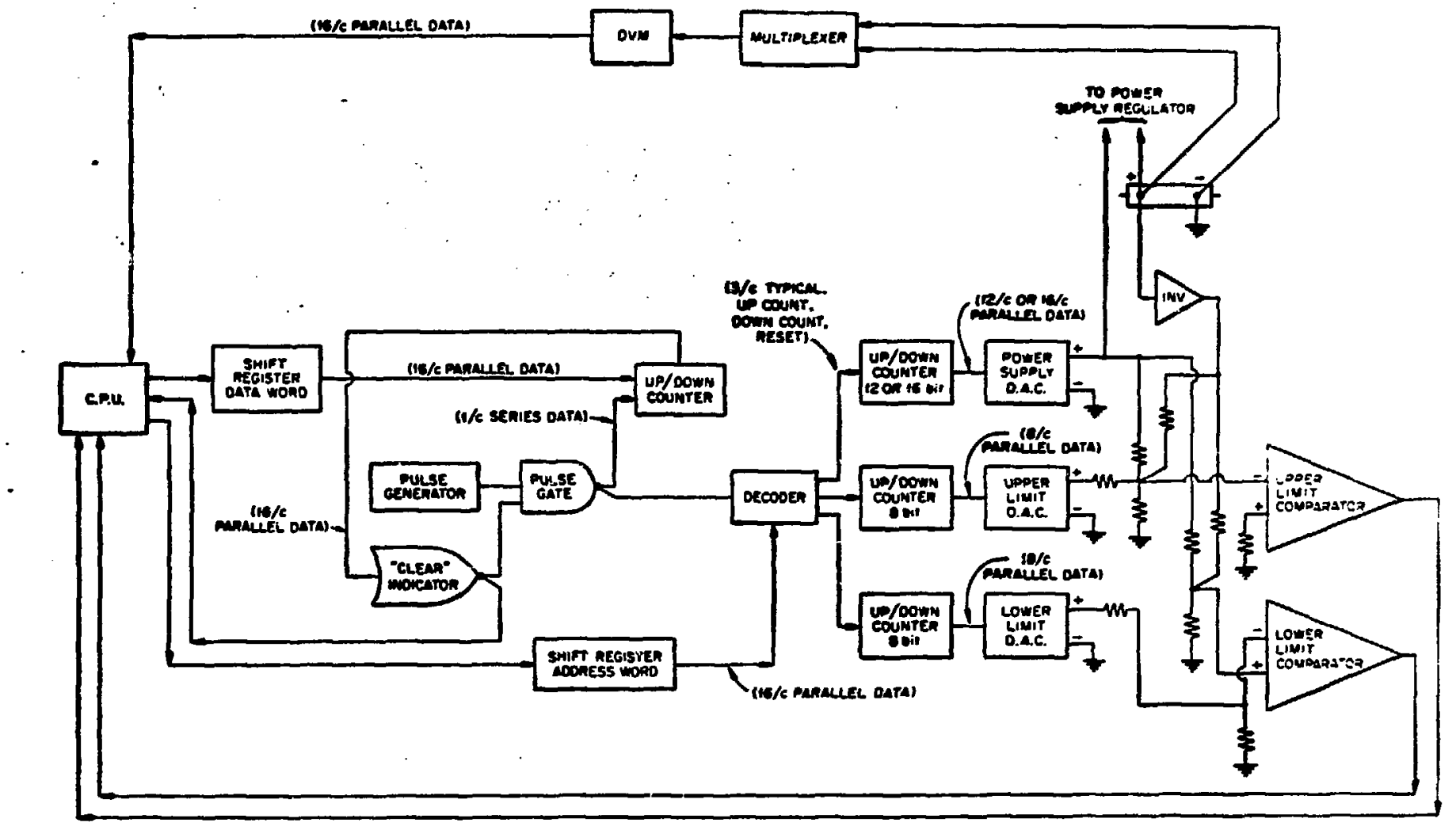

F18. I A typical power supply control loop Incorporating DAC's and comparators. Additional power supplies are controlled by appropriate dat 3 branching from the decoder. 


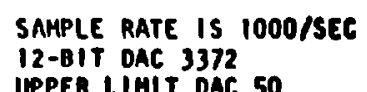

12-BIT DAC 3372

UPPER LIAIT DAC 50

LOMER LIMIT DAC 50
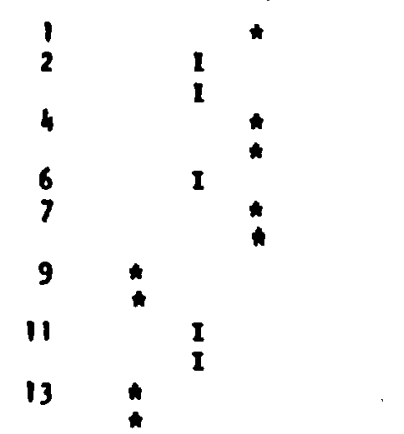

$15 \quad I$
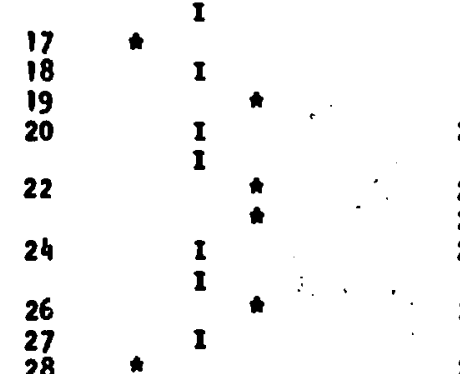

29 I
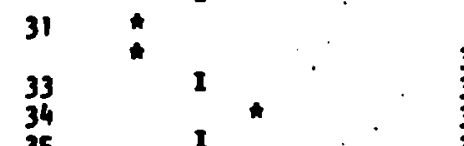

351

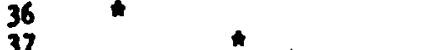

38

40

42.1

43
45
46
47

49

52 *

57
SAMPLE RATE is 1000/SEC

12-8IT DAC 3372

UPPER LIAIT DAC 100

LOMER LIMIT DAC 100
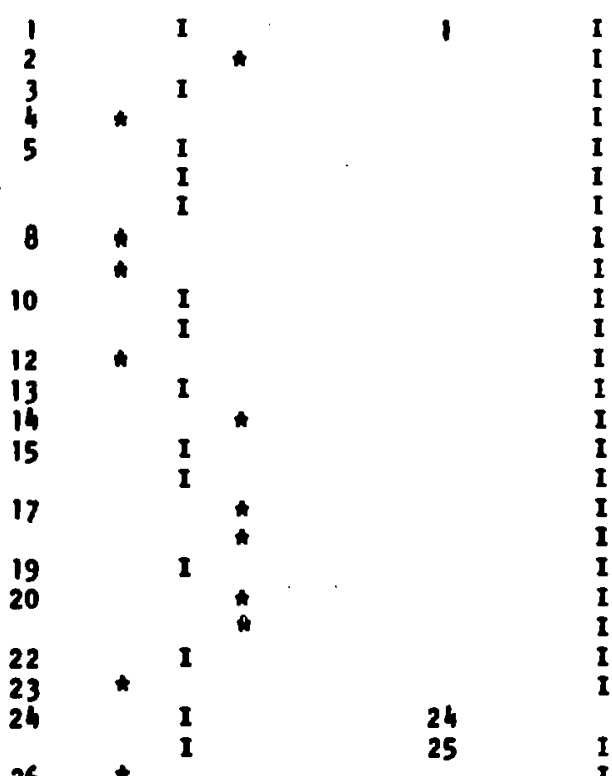

27
28

28
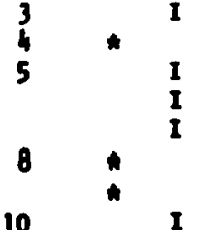

10 I

$12 *$

14

$15 \quad I$

17

19

20

22 - I

24 I

26 .

.

SAMPLE RATE IS 1000/SEC

12-BIT DAC 3372

UPPER LIMIT DAC 225

LOMER LIMIT OAC 225
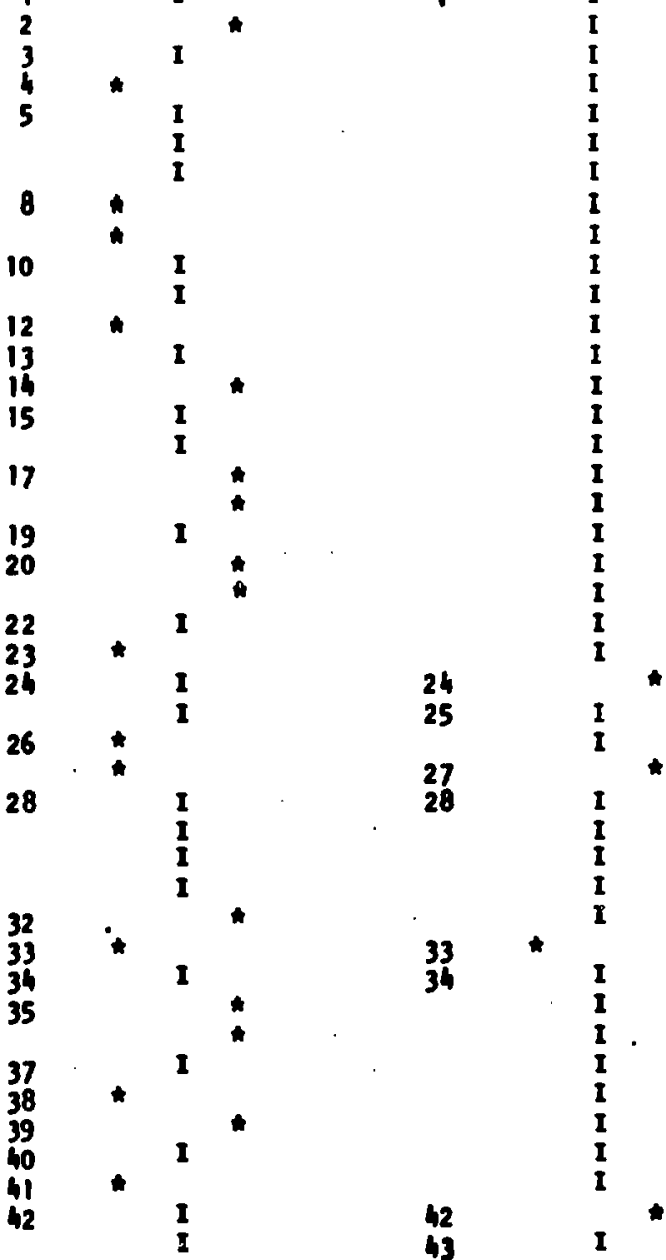

$\begin{array}{llll}44 & * & & \\ 46 & & 1 & \\ 47 & & & \\ 48 & & 1 & \\ 50 & & 1 & \\ 51 & & 1 & \\ & & 1 & \\ 54 & & 1 & \\ 55 & & 1 & \\ 57 & & 1 & \\ 58 & & 1 & \end{array}$

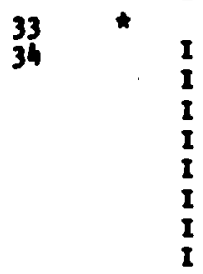

42

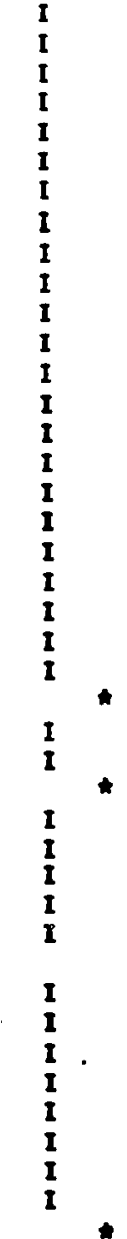

$\mathbf{I}$
$\mathbf{I}$
$\mathbf{I}$
$\mathbf{I}$
$\mathbf{I}$
$\mathbf{I}$
$\mathbf{I}$
$\mathbf{I}$
$\mathbf{I}$
$\mathbf{I}$
$\mathbf{I}$
$\mathbf{I}$
$\mathbf{I}$
$\mathbf{I}$
$\mathbf{I}$
$\mathbf{I}$

718. 2 . Print-oute for three aeta of computer scans of the 11mit DAC's for varlous tolerance settinge. Blasped time in millisecondo from the first scan in esch wet is shown on the left of the comparator state. 